\title{
The spectrum, incidence, kinetics and management of endocrinopathies with immune checkpoint inhibitors for metastatic melanoma
}

\author{
Emma S Scott ${ }^{1,2}$, Georgina V Long ${ }^{2,3,4}$, Alexander Guminski ${ }^{2,3,4}$, Roderick J Clifton-Bligh ${ }^{1,2}$, \\ Alexander M Menzies ${ }^{2,3,4, *}$ and Venessa H Tsang ${ }^{1,2, *}$ \\ ${ }^{1}$ Department of Endocrinology, Royal North Shore Hospital, Sydney, Australia, ${ }^{2}$ Sydney Medical School, The \\ University of Sydney, Sydney, Australia, ${ }^{3}$ Melanoma Institute Australia, Sydney, Australia, and ${ }^{4}$ Department of \\ Medical Oncology, Royal North Shore and Mater Hospitals, Sydney, Australia \\ *(A M Menzies and V H Tsang contributed equally to this work)
}

Correspondence should be addressed to V Tsang

Email

Venessa.tsang@sydney.edu.au

\begin{abstract}
Objective: Endocrine immune-related adverse events (endocrinopathies) are increasingly prevalent with the use of immune checkpoint inhibitors for the treatment of metastatic melanoma and other malignancies. There are no evidence-based guidelines for the screening or management of such patients. To describe the spectrum, incidence, kinetics and management of endocrinopathies with immune checkpoint inhibitors.
\end{abstract}

Design: A prospective study conducted at Melanoma Institute Australia between April 2014 and October 2015.

Methods: A total of 177 patients were treated with (a) ipilimumab $(n=15)$, (b) anti-PD-1 (nivolumab, pembrolizumab) $(n=103)$ or (c) combination ipilimumab and anti-PD-1 $(n=59)$ and were screened and managed for the subsequent endocrinopathies. The main outcome measures were the incidence and kinetics of endocrinopathy by immunotherapy drug class.

Results: Thirty-one patients (18\%) developed an endocrine immune-related adverse event (thyroid dysfunction: $14 \%$, hypophysitis: $6 \%$ and autoimmune diabetes: $0.6 \%$ ). Combination immunotherapy was more likely to result in a single or multiple endocrinopathy compared to anti-PD-1 monotherapy ( $27 \%$ vs $9 \%$ and $7 \%$ vs $0 \%$ respectively, $P<0.01$ ). Endocrinopathies occurred after a median of 8 weeks from treatment commencement (range: 12-225 days), with combination immunotherapy resulting in significantly earlier onset compared to ipilimumab (median: 30 vs 76 days, $P=0.046$ ). The majority of endocrinopathies were identified in asymptomatic patients with hormonal screening. There were no baseline predictors for endocrinopathy.

Conclusions: Combination immunotherapy has a greater risk of development of endocrinopathy compared to anti-PD-1 monotherapy. Regular biochemical profiling of patients, particularly within the first twelve weeks, results in early detection of endocrinopathy to minimise morbidity.

\section{Introduction}

Drugs that reactivate the immune system have transformed the therapeutic landscape in oncology. Antibodies that block the PD-1/PD-L1 (nivolumab, pembrolizumab, atezolizumab, durvalumab) and CTLA-4 (ipilimumab) (c) 2018 European Society of Endocrinology Printed in Great Britain checkpoints remove inhibition of $\mathrm{T}$ cells and unleash $\mathrm{T}$ cell-mediated anti-tumour responses, resulting in durable responses and long-term survival in cancer patients $(1,2,3,4,5)$. Anti-PD-1/PD-L1 therapies are now approved 
for many cancers including melanoma, non-smallcell lung cancer, renal cell carcinoma, bladder cancer, head and neck squamous cell carcinoma and Hodgkin's lymphoma, while ipilimumab is approved in melanoma, as monotherapy and in combination with nivolumab.

While immune checkpoint inhibitors have impressive activity, they cause a vast range of immune-related adverse events (irAEs), and the endocrine system is often involved. The most frequent ipilimumab-induced endocrinopathy is hypophysitis, occurring in as many as $17 \%$ of patients in retrospective series $(2,6,7,8)$, while thyroiditis (3\%), hypothyroidism (6\%) and adrenal insufficiency (1\%) also rarely occur (8). Anti-PD-1/PD-L1 antibodies have less overall toxicity than ipilimumab, and they also have a different endocrinopathy profile, with less hypophysitis (1-6\%), but more frequent thyroiditis $(6.1 \%)$ and hypothyroidism $(9 \%)(5,9)$. Toxicity with the combination of ipilimumab and anti-PD-1 occurs more frequently than either administered as monotherapy, with thyroid dysfunction reported to occur in up to $25 \%$ of subjects and hypophysitis in up to $11.7 \%$ in randomised controlled trial data $(3,10,11)$. As yet, there is limited data available and little is known about the kinetics of endocrinopathies associated with combination immunotherapy.

Immune-related endocrinopathies are common and usually mild, but require vigilance and prompt management to prevent significant morbidity. To date, there are no predictors for who will develop endocrine toxicity. In addition, there are no evidenced-based guidelines as to how patients should be monitored during therapy or how endocrinopathies should be best managed. The aim of this study was to characterise the spectrum, incidence, kinetics and management of endocrinopathies with immune checkpoint inhibitors.

\section{Subjects and methods}

\section{Patients}

All patients with metastatic melanoma treated with immunotherapy between April 2014 and October 2015 at Melanoma Institute Australia were included for analysis. Patients were followed to 31st December 2015. Treatments included anti-CTLA-4 (ipilimumab) or anti-PD-1 antibodies (nivolumab or pembrolizumab), either as monotherapy or in combination $(3 \mathrm{mg} / \mathrm{kg}$ ipilimumab with $1 \mathrm{mg} / \mathrm{kg}$ nivolumab or $1 \mathrm{mg} / \mathrm{kg}$ ipilimumab with $2 \mathrm{mg} / \mathrm{kg}$ pembrolizumab). Patients who received sequential ipilimumab and then anti-PD-1 therapy (or vice versa) were excluded from analysis due to the varying intervals between lines of treatment, and the potential for prior endocrinopathies from 1st-line therapy to influence later toxicity, including the use of steroids to manage endocrinopathies (or other toxicities) at commencement of second-line therapy. All patients with clinical or biochemical endocrine abnormalities were referred to a single endocrinologist. Baseline clinicopathologic data, treatment and outcome data were examined.

\section{Endocrine assessments}

All patients underwent hormone assessment profiling prior to commencement of immunotherapy and prior to each subsequent cycle, three weekly for pembrolizumab, ipilimumab and combination therapy, and second weekly for nivolumab, for at least the first twelve weeks of treatment. Assessment included measurement of thyrotropin-stimulating hormone (TSH), thyroxine (T4) and cortisol. If any abnormality was detected, a complete pituitary profile (cortisol, ACTH, TSH, T4, T3, IGF-1, GH, FSH, LH, testosterone, oestradiol and prolactin) and thyroid autoantibodies (anti-thyroglobulin (Tg), anti-thyroid peroxidase (TPO) and thyroidstimulating hormone receptor antibody (TRAB)) were assessed. Hypophysitis was defined as secondary adrenal insufficiency (low serum cortisol in absence of exogenous steroid \pm symptoms and consistent MRI pituitary findings), secondary hypothyroidism or secondary hypogonadism. Data regarding the incidence, kinetics of onset and offset and treatment of endocrinopathies were determined. The study was undertaken with institutional Human Ethics Review Committee approval.

\section{Statistical analysis}

IBM SPSS Statistics (version 22) and Graph Pad Prism (version 6.0) were used for data analyses, including descriptive statistics, independent $T$-tests, Kruskal-Wallis tests, ANOVA or chi-squared. Post hoc analysis occurred with adjustment for multiple comparisons via Bonferroni or Dunnett's test. Statistical significance was taken at $P<0.05$.

\section{Results}

\section{Baseline characteristics}

One hundred and seventy-seven patients were examined (Table 1). The median age at treatment commencement 
Table 1 Characteristics of patients who developed an endocrine immune-related adverse event during the study period. Values are presented as $n(\%)$ or median (interquartile range). $P$ value comparison across immunotherapy groups.

\begin{tabular}{|c|c|c|c|c|c|}
\hline & Ipilimumab & Anti-PD-1 monotherapy & Combination & Total & $P$ value \\
\hline Patients, $n$ & 15 & 103 & 59 & 177 & - \\
\hline Median age (years) & $70.0(9.2)$ & $68.3(26.3)$ & $64.5(15.9)^{\mathrm{a}, \mathrm{b}}$ & $67.8(19.5)$ & 0.02 \\
\hline Males, $n(\%)$ & $13(87)$ & $60(58)$ & $42(71)$ & $115(65)$ & 0.046 \\
\hline Endocrinopathy, $n(\%)$ & $6(40)$ & $9(9)^{c, d}$ & $16(27)$ & $31(18)$ & 0.001 \\
\hline Hypophysitis, $n(\%)$ & $5(33)$ & $0^{e, f}$ & $6(10)$ & $11(6)$ & $<0.001$ \\
\hline Thyroid dysfunction, $n(\%)$ & $2(13)$ & $9(9)$ & $13(22)$ & $22(14)$ & 0.06 \\
\hline Autoimmune diabetes, $n(\%)$ & 0 & 0 & $1(2)$ & $1(0.6)$ & 0.4 \\
\hline Multiple endocrinopathies, $n(\%)$ & $1(7)$ & $0^{9}$ & $4(7)$ & $5(3)$ & 0.03 \\
\hline
\end{tabular}

Post hoc analysis: aipilimumab vs combination $P=0.007$, banti-PD- 1 vs combination $P=0.02$, canti-PD- 1 vs ipilimumab $P=0.0007$, danti-PD- 1 vs combination $P=0.0018$, eanti-PD-1 vs ipilimumab $P<0.0001$, fanti-PD-1 vs combination $P=0.001$, 9anti-PD-1 vs combination $P=0.0075$.

was 67.8 years, and $65 \%$ were male. Most patients received anti-PD-1 therapy $(n=103), 59$ received combination ipilimumab and anti-PD-1 therapy (five: ipilimumab and nivolumab, 54: ipilimumab and pembrolizumab) and fifteen received ipilimumab monotherapy.

\section{Incidence and spectrum of endocrinopathies}

Endocrinopathies developed in 31 (18\%) patients; 40\% with ipilimumab, 9\% with anti-PD-1 therapy and $27 \%$ with combination therapy (Table 1). Individuals treated with anti-PD-1 monotherapy were less likely to develop an endocrinopathy than ipilimumab or combination groups $(P<0.01$, respectively). Anti-PD-1 monotherapy was also less likely to result in multiple endocrinopathies than combination treatment $(P<0.01)$. Overall, thyroid dysfunction occurred in 14\%, hypophysitis in $6 \%$ and autoimmune diabetes in $0.6 \%$. Thyroid dysfunction developed in $13 \%$ patients with ipilimumab, 9\% patients with anti-PD- 1 and $22 \%$ of patients treated with combination therapy $(P=0.06)$. Hypophysitis developed in $33 \%$ patients with ipilimumab, $22 \%$ of patients with combination therapy and no patients with anti-PD-1 monotherapy $(P<0.001)$. One patient $(0.5 \%)$ developed type 1 diabetes, and multiple endocrinopathies occurred in $5(3 \%)$ patients. Sex and age were not associated with the development of endocrinopathy ( $P>0.05$, respectively).

\section{Kinetics of endocrinopathies}

There was a significant difference in the time from drug commencement to development of any endocrinopathy by drug group, combination therapy (median 30 \pm 113 days), ipilimumab (76 156 days) and anti-PD-1 therapies (67 \pm 190 days) $(P=0.02)$ (Fig. 1A). Combination therapy resulted in significantly faster onset to endocrinopathy compared to ipilimumab $(P=0.046)$.
There was a difference in the time to development of thyroid dysfunction by treatment, with combination therapy (median: $30 \pm 113$ days) developing faster than anti-PD-1 therapy (median: $65 \pm 190$ days) or ipilimumab (median: $123 \pm 122$ days) $(P=0.043)$ (Fig. 1B). However, no difference was observed between the drug groups in the post hoc analysis. There was no difference in the median time from drug commencement to onset of hypophysitis in patients treated with ipilimumab (median: $83 \pm 129$ days) or combination (median: $71 \pm 146$ days) $(P=0.54)$ (Fig. 1C). Of the 9 patients whose thyroid dysfunction resolved, there was no difference in the median time to
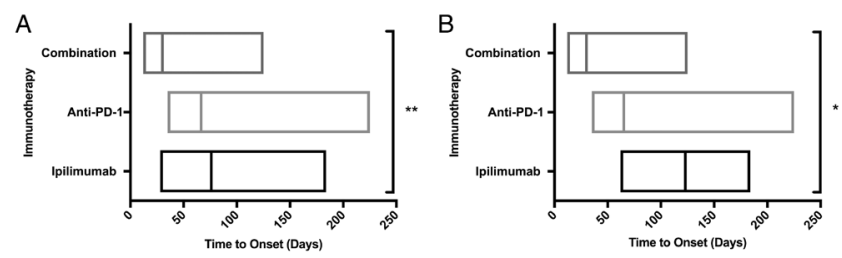

C

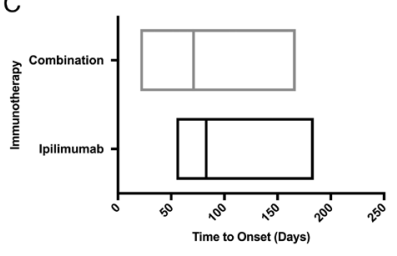

D

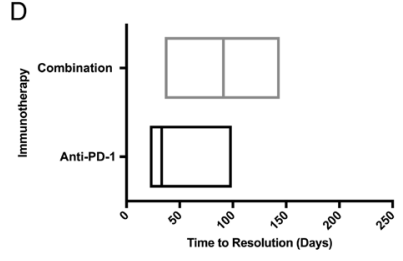

\section{Figure 1}

(A) Time from drug commencement to development of any endocrinopathy. (B) Time from drug commencement to onset of thyroid dysfunction. (C) Time from drug commencement to onset of hypophysitis. (D) Time to resolution of hyperthyroidism or subclinical hyperthyroidism to euthyroidism. The values are for those patients' whose thyroid dysfunction resolved. Values are range and the line marks the median. ${ }^{*} P<0.05,{ }^{*} P<0.005$. Combination, ipilimumab and anti-PD-1. 
resolution in those treated with anti-PD-1 (33 \pm 77 days) or combination therapy $(91 \pm 108$ days $)(P=0.17)$ (Fig. 1D).

\section{Thyroid dysfunction}

\section{Presentation, treatment, resolution}

Thyroid dysfunction occurred in 24 patients and was diagnosed on routine screening while asymptomatic in the vast majority $(n=19,79 \%)$. All the patients diagnosed symptomatically were hyperthyroid. Most patients $(n=21,88 \%)$ were frankly or subclinically hyperthyroid at diagnosis, which then evolved to hypothyroidism $\quad(n=9,38 \%)$, euthyroidism $\quad(n=9$, $38 \%)$ or remained subclinically hyperthyroid $(n=2$, $8 \%)$ or frankly hyperthyroid $(n=1,4 \%)$ over the study period. The median time taken for hyperthyroidism or

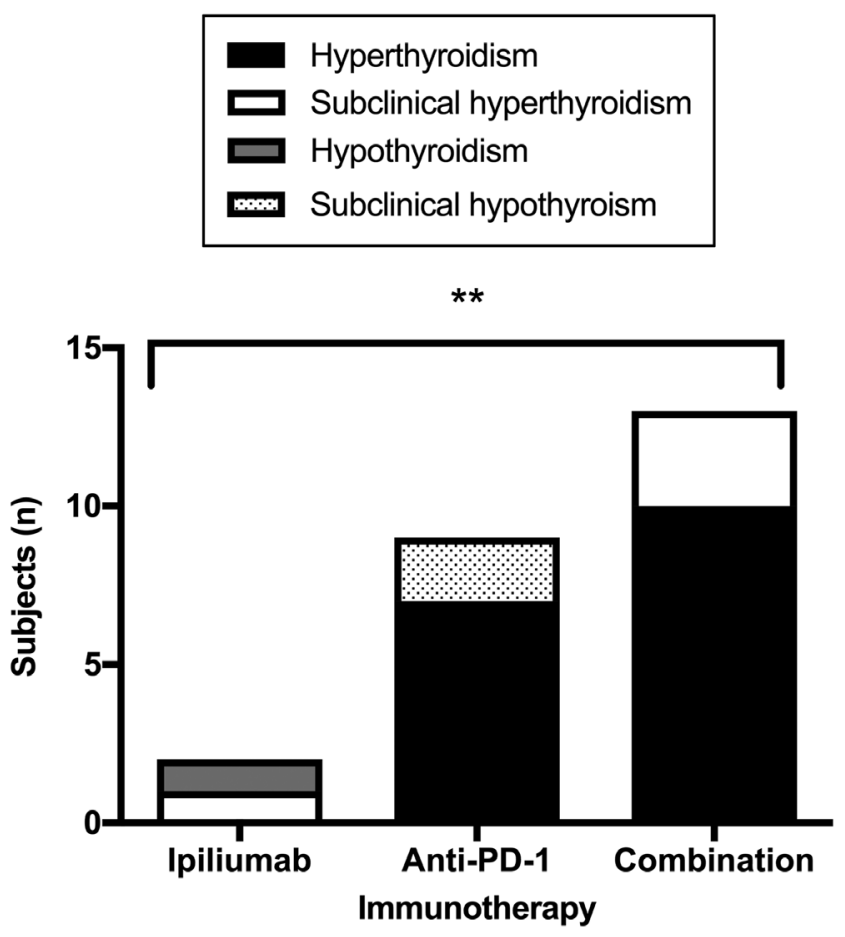

Figure 2

Biochemical presentation of thyroid dysfunction by immunotherapy group. 7 (29\%) and 10 (42\%) in the anti-PD-1 and combination group presented with hyperthyroidism, 1 $(4 \%)$ and $3(13 \%)$ in the ipilimumab and combination group presented with subclinical hyperthyroidism, 1 (4\%) patient from the ipilimumab group presented with hypothyroidism and $2(8 \%)$ anti-PD-1 patients presented with subclinical hypothyroidism $(P=0.004)$. Percentage of patients who developed thyroid dysfunction. subclinical hyperthyroidism to evolve to hypothyroidism was $37 \pm 50$ days. Of note, the follow-up time in the one individual whose hyperthyroidism persisted was only 12 days. Three (13\%) of patients were hypothyroid or had subclinical hypothyroidism at diagnosis. The biochemical presentation of thyroid dysfunction significantly differed by immunotherapy group (Fig. 2). Seven (29\%) individuals treated with anti-PD-1 and ten $(42 \%)$ with combination immunotherapy presented with hyperthyroidism, whereas no patients in the ipilimumab arm presented with frank hyperthyroidism. Seven (29\%) patients with thyroid dysfunction had the following positive thyroid autoantibodies: anti-TPO and anti-Tg $(n=2,8 \%)$, anti-TPO $(n=2,8 \%)$, anti-Tg $(n=2,8 \%)$, anti-TPO and TRAB $(n=1,4 \%)$. There was no difference in the resolution of thyroid dysfunction by thyroid autoantibody status $(P=0.06)$.

Patients who developed hyperthyroidism were only commenced on anti-thyroid drugs (ATDs) or beta blockade if they were symptomatic and if free $\mathrm{T} 4$ exceeded $30 \mathrm{pmol} / \mathrm{L}$. Of the 21 patients with hyperthyroidism, four (19\%) required treatment with ATDs and beta blockade, five (24\%) required beta blockade alone and twelve (57\%) required no treatment. There was no difference in the requirement for ATDs by immunotherapy $(P=0.6)$. The use of ATDs did not affect the pattern or median time to resolution of hyperthyroidism ( $50 \pm 21$ days with ATDs and $40 \pm 122$ days without, $P>0.05$ ). A total of 12 patients (7\%) developed hypothyroidism and required thyroxine replacement, including nine $(38 \%)$ of those who initially presented with hyperthyroidism. All patients who presented with hypothyroidism or subclinical hypothyroidism required ongoing treatment with thyroxine.

\section{Hypophysitis}

Presentation, treatment, resolution

Eleven patients were diagnosed with hypophysitis, five (45\%) while asymptomatic and by routine biochemical screening. Amongst patients treated with combination immunotherapy, five (83\%) presented symptomatically. At the time of symptomatic presentation, three patients initially had a normal hormonal screen that subsequently became abnormal, despite administering high-dose steroids in one of these individuals. Patients who presented symptomatically reported a combination of fatigue, headache, myalgia, nausea or visual changes. One patient presented with adrenal crisis (hypotension, nausea, vomiting, hyponatraemia). Those treated with combination therapy were more likely 
to have a symptomatic presentation $(n=5,83 \%)$ than those treated with ipilimumab $(n=1,20 \%)(P=0.036)$. Seven $(64 \%)$ patients had adrenal (A), gonad $(\mathrm{G})$ and thyroid (T) axes involved, while $A / T, A / G, A$ only and $G$ only occurred in one patient each. There was no significant difference in the hormonal axes affected in the ipilimumab or combination groups, nor in the time to onset between affected axes. Ten patients underwent MRI brain a median of $4.5 \pm 63$ days following a diagnosis of hypophysitis, three patients had consistent radiological findings (bulky pituitary, thickening and abnormal enhancement of infundibulum, normal posterior bright spot) and one patient had partial empty sella. No cases of secondary adrenal insufficiency or secondary hypothyroidism normalised, but secondary hypogonadism resolved without treatment in three $(27 \%)$ patients. Patients were treated with physiologic doses of steroid replacement (5-7.5 mg prednisone), unless supraphysiological doses were otherwise needed for stress dosing or for other immune-related adverse events as occurred in two patients. Testosterone supplementation was administered topically or via intra-muscular injection. Thyroid replacement was performed as per standard practice.

\section{Autoimmune diabetes}

A 58-year-old male treated with combination ipilimumab and pembrolizumab developed autoimmune diabetes. Twelve days after commencement of immunotherapy subclinical hyperthyroidism was diagnosed on routine screening, with negative thyroid autoantibodies, and this was monitored. Pituitary profile was unremarkable. He later presented with diabetic ketoacidosis sixty-two days after immunotherapy commencement. On admission, blood gas $\mathrm{pH}$ was 7.29 (7.35-7.45), bicarbonate $21 \mathrm{mmol} / \mathrm{L}$ (25-31) and glucose $36.7 \mathrm{mmol} / \mathrm{L}$ (3.5-7.8). Anti-glutamate decarboxylase antibody $<5 \mathrm{IU} / \mathrm{mL}(<10)$, anti-IA2 antibody $<10(<10)$, islet-cell antibody-negative and insulin antibodies $<0.4 \mathrm{kU} / \mathrm{L}(<0.4)$ were negative. In addition, coeliac serology was negative. Glycated haemoglobin (HbA1c) at presentation was 6.8\%. The patient required treatment with intravenous fluids and insulin and has subsequently been stabilised with subcutaneous insulin.

\section{Relationship between endocrinopathies and other toxicities and outcome}

Sixteen (52\%) patients who developed an endocrinopathy had a RECIST response to treatment, while 11 (32\%) had stable disease and five (16\%) had progressive disease. Eight (25\%) patients with an endocrinopathy had previously been treated with head or neck radiotherapy. One patient who developed antibody-negative thyroiditis had a background of pre-existing Graves' disease. Twenty (65\%) patients who developed an endocrinopathy also developed another immune-related adverse event of varying grades including colitis, pneumonitis, hepatitis and dermatitis.

\section{Discussion}

Immune checkpoint inhibitors are increasingly used in the clinic, and hence, endocrinopathies are becoming more prevalent in the community, posing a challenge to oncologists, endocrinologists and general practitioners alike. Combination immunotherapy with ipilimumab and nivolumab is now standard care for a large proportion of patients with melanoma. To the authors' knowledge, this is the first study to examine the spectrum, incidence, kinetics and management of endocrinopathies with immune checkpoint inhibitors in detail, particularly those on combination therapy. Results of this study suggest that up to one quarter of patients treated with combination immunotherapy develop endocrinopathies and that they occur more frequently and earlier than with anti-PD-1 monotherapy, usually with 5 weeks of treatment commencement. Routine biochemical screening (prior to each infusion in the first twelve weeks) detects most endocrinopathies before they become symptomatic, resulting in earlier treatment and less morbidity.

Most endocrinopathies occurred within 8 weeks of immunotherapy commencement, earlier with combination immunotherapy than anti-PD-1 therapy. Previous reports have described the time to onset of any endocrinopathies, between 9 and 12 weeks post initiation of anti-CTLA-4 $(12,13)$ and following $4-18$ weeks after anti-PD-1 therapy $(14,15)$, with a recent retrospective pooled analysis of 576 patients on nivolumab trials reporting a median 10.4 weeks to endocrinopathy onset (15). The time course to development of endocrinopathies with combination immunotherapy has not been detailed previously. This has implications for the clinical management and duration of screening for these patients. Individuals treated with combination immunotherapy may require more frequent endocrine profiling than those treated with anti-PD-1 monotherapy. Combination therapy was associated with a greater incidence of single 
and multiple endocrinopathies than anti-PD-1 therapy, consistent with previous research (15). Combination immunotherapy resulted in a full spectrum of endocrine side effects, including the rare but reported autoimmune diabetes, which is thought to be associated with PD-1 blockade (16). Anti-PD-L1 agents were not assessed in this study; however, they may have behaved differently to anti-PD-1 therapies (17). Anti-PD-L1 is associated with the development of autoimmune diabetes; however, the spectrum of endocrinopathies with these therapies require further study (18). In contrast to our study, other groups have reported ipilimumab monotherapy to result in a lower rate of endocrine immune adverse events relative to combination dual-therapy $(3,10)$. The lack of difference observed in the incidence of endocrine immune-related adverse events between ipilimumab and combination groups is likely due to the small numbers receiving ipilimumab therapy.

The vast majority of patients in this study were diagnosed with thyroid dysfunction on routine screening; however, a minority of hyperthyroid individuals were diagnosed symptomatically and required anti-thyroid therapy. Biochemical presentation of thyroid dysfunction differed by immunotherapy, with all individuals treated with combination immunotherapy presenting with frank or subclinical hyperthyroidism. There was no difference in the resolution of thyroid dysfunction or need for treatment by immunotherapy group, and the use of ATDs did not alter subsequent development of hypothyroidism. Approximately one-third of patients with thyroid dysfunction had positive thyroid autoantibodies at diagnosis, consistent with a recent study assessing thyroid immune-related adverse events with pembrolizumab (15). Anti-thyroid peroxidase antibodies are detected in $8.6 \%$ of the Australian population (19), suggesting an association for increased risk of the development of thyroid dysfunction with immunotherapy in the presence of thyroid antibodies. Baseline thyroid autoantibodies were not available in our cohort and should be examined in future studies.

Most patients (55\%) with hypophysitis presented with symptoms, particularly in those treated with combination immunotherapy. In keeping with other studies, most patients had non-specific symptoms including nausea, fatigue or headache with only one classical presentation of adrenal crisis (20). Our study reported higher rates of asymptomatic diagnoses (45\%) than described previously $(8,21,22)$, which may be due to the universal regular profiling of our study cohort, resulting in earlier detection of early-onset hypophysitis. This highlights the importance of routine screening over the 12 weeks when hypophysitis is most likely to develop. Of the subset of patients who underwent radiological imaging at the time of diagnosis of hypophysitis, 30\% had radiological signs in keeping with hypophysitis $(8,21,23,24)$. Previous groups have highlighted the rapid resolution of radiographic changes; however, pituitary imaging may help to exclude differential diagnoses such as metastases or pituitary adenoma $(25,26)$. The hormonal deficiencies described are similar to that seen elsewhere, with the most common deficiencies being adrenal insufficiency and hypothyroidism $(8,27)$. Two patients were administered stress doses of glucocorticoids in this study; however, no cases of secondary adrenal insufficiency or secondary hypothyroidism recovered. The role of high-dose steroids has been discussed elsewhere, and it does not appear to alter the natural history or progression of hypophysitis (23, 28 ). None of the patients in the study required cessation of immunotherapy due to endocrinopathy. Previous authors have proposed that the development of an endocrinopathy should not prompt withholding immunotherapy as the therapeutic advantages of these drugs counterbalance any such side effects (22). Other studies have reported minimal recovery of the corticotrophic axes, while thyroid and hypogonadal dysfunction may recover $(6,23)$. Recovery of thyroid or gonadal dysfunction may be as a result of sick hypogonadism or sick euthyroidism (6). As a result, some authors propose delay of commencement of sex hormones.

The strengths of this study include the consecutive cohort of patients, longitudinal biochemical profiling of patients throughout therapy, referral for all patients with abnormalities to a specialist endocrinologist, and the exclusion of patients that sequenced between immunotherapies, which may bias toxicity development. Limitations include the small number of patients treated with ipilimumab monotherapy; however, this treatment is no longer a standard of care in melanoma, at least in the first-line setting. Patients did not undergo baseline autoantibody profiling, which may have detected pre-existing occult endocrine disease, particularly thyroid disease. It is the authors' recommendation that routine endocrine profiling should occur prior to each administration of immunotherapy for the first 12 weeks of treatment. Subsequent screening with TSH measurements should occur less frequently (6-12 weekly) and other profiling should be guided by symptoms. 
This study is the first detailed description of the endocrine immune-related adverse events associated with anti-PD-1 antibody therapy, alone and in combination with ipilimumab. Endocrinopathies are common and often occur early, especially in those on combination therapy, can often be detected biochemically before overt symptoms develop and require specific therapy. Further research is required to determine those at risk of endocrine toxicity, and immunotherapy-induced endocrinopathies may offer insights into the pathogenesis of idiopathic endocrine autoimmunity.

\section{Declaration of interest}

On behalf of myself and all co-authors, I accept the terms of the copyright transfer/affirmation of originality. E S, no conflicts of interest in this work. G L, consultant advisor to Amgen, Array, BMS, MERCK MSD, Novartis, Pierre Fabre, Roche. A G, no conflicts of interest relevant to this work. $R$ C B, advisory board: Eisai, Bayer, Amgen and Lilly and speaker fees from Eisai, Amgen, Ipsen and Lilly. There are no conflicts of interest relevant to this work. A M M, advisory board: Novartis, MSD, Chugai, Pierre Fabre. Honoraria; BMS, Roche. V T, no conflicts of interest in this work.

\section{Funding}

This research did not receive any specific grant from any funding agency in the public, commercial or not-for-profit sector.

\section{Author contribution statement}

E S contributed to data collection, analysis and manuscript. V T contributed to data collection and manuscript. A M contributed to manuscript. G L contributed to manuscript.

\section{Acknowledgements}

Authors thank all patients, clinicians, scientists and administration team members who have contributed to this research.

\section{References}

1 Postow MA, Callahan MK \& Wolchok JD. Immune checkpoint blockade in cancer therapy. Journal of Clinical Oncology 201533 1974-1982. (https://doi.org/10.1200/JCO.2014.59.4358)

2 Hodi F, O'Day S, McDermott D, Weber R, Sosman J, Haanen J, Gonzalez R, Robert C, Schadendorf D, Hassel J et al. Improved survival with Ipilimumab in patients with metastatic melanoma. New England Journal of Medicine 2010363 711-723. (https://doi. org/10.1056/NEJMoa1003466)

3 Larkin J, Chiarion-Sileni V, Gonzalez R, Grob J, Cowey C, Lao C, Schadendorf D, Dummer R, Smylie M, Rutkowski P et al. Combined Nivolumab and Ipilimumab or monotherapy in untreated melanoma. New England Journal of Medicine 2015373 23-34. (https:// doi.org/10.1056/NEJMoa1504030)

4 Robert C, Long G, Brady B, Dutriaux C, Maio M, Mortier L, Hassel J, Rutkowski P, McNeil C, Kalinka-Warzocha E et al. Nivolumab in previously untreated melanoma without BRAF mutation. New England Journal of Medicine 2015372 320-330. (https://doi. org/10.1056/NEJMoa1412082
5 Robert C, Schachter J, Long G, Arance A, Grob J, Mortier L, Daud A, Carlino M, McNeil C, Lotem M et al. Pembrolizumab versus Ipilimumab in advanced melanoma. New England Journal of Medicine 2015372 2521-2532. (https://doi.org/10.1056/NEJMoa1503093)

6 Corsello S, Barnabei A, Marchetti P, De Vecchis L, Salvatori R \& Torino F. Endocrine side effects induced by immune checkpoint inhibitors. Journal of Clinical Endocrinology and Metabolism 201398 1361-1375. (https://doi.org/10.1210/jc.2012-4075)

7 Torino F, Barnabei A, De Vecchis L, Salvatori R \& Corsello S. Hypophysitis induced by monoclonal antibodies to Cytotoxic T Lymphocyte Antigen 4: challenges from a new cause of a rare disease. Oncologist 201217 525-535. (https://doi.org/10.1634/ theoncologist.2011-0404)

8 Ryder M, Callahan M, Postow M, Wolchok J \& Fagin J. Endocrinerelated adverse events following ipilimumab in patients with advanced melanoma: a comprehensive retrospective review from a single institution. Endocrine-Related Cancer 201421 371-381. (https:// doi.org/10.1530/ERC-13-0499)

9 Naidoo J, Page D, Li B, Connell L, Schindler K, Lacouture M, Postow M \& Wolchok J. Toxicities of the anti-PD-1 and anti-PD-L1 immune check point antibodies. Annals of Oncology 201526 2375-2391. (https://doi.org/10.1093/annonc/mdv383)

10 Postow M, Chesney J, Pavlick A, Robert C, Grossmann K, McDermott D, Linette G, Meyer N, Giguere J, Agarwala S et al. Nivolumab and Ipilimumab versus Ipilimumab in untreated melanoma. New England Journal of Medicine 2015372 2006-2017. (https://doi.org/10.1056/NEJMoa1414428)

11 Long GV, Atkinson V, Cebon JS, Jameson MB, Fitzharris BM, McNeil CM, Hill AG, Ribas A, Atkins MB \& Thompson JA. Pembrolizumab (pembro) plus ipilimumab (ipi) for advanced melanoma: results of the KEYNOTE-029 expansion cohort. Oral Abstract. Presented at the 2016 Annual Meeting of the American Society of Clinical Oncology.

12 Weber J, Kahler K \& Hauschild A. Management of immune-related adverse events and kinetics of response with ipilimumab. Journal of Clinical Oncology 201230 2691-2697. (https://doi.org/10.1200/ JCO.2012.41.6750)

13 Weber J, Dummer R, de Pril V, Lebbe C \& Hodi F. Patterns of onset and resolution of immune-related adverse events of special interest with ipilimumab: detailed safety analysis from a phase 3 trial in patients with advanced melanoma. Cancer 2013119 1675-1682. (https://doi.org/10.1002/cncr.27969)

14 Eigentler T, Hassel J, Berking C, Aberle J, Bachmann O, Grunwald V, Kahler K, Loquai C, Reinmuth N, Steins M et al. Diagnosis, monitoring and management of immune-related adverse drug reactions of anti-PD-1 antibody therapy. Cancer Treatment Reviews 201645 7-18. (https://doi.org/10.1016/j.ctrv.2016.02.003)

15 Weber JS, Hodi FS, Wolchok JD, Topalian SL, Schadendorf D, Larkin J, Sznol M, Long GV, Li H, Waxman IM et al. Safety profile of Nivolumab monotherapy: a pooled analysis of patients with advanced melanoma. Journal of Clinical Oncology 201735 785-792. (https://doi.org/10.1200/JCO.2015.66.1389)

16 Hughes J, Vudattu N, Sznol M, Gettinger S, Kluger H, Lupsa B \& Herold K. Precipitation of autoimmune diabetes with anti-PD-1 immunotherapy. Diabetes Care 201538 55-57. (https://doi. org/10.2337/dc14-2349)

17 Topalian SL, Drake CG \& Pardoll DM. Targeting the PD-1/ B7-H1(PD-L1) pathway to activate anti-tumor immunity. Current Opinion in Immunology 201224 207-212. (https://doi.org/10.1016/j. coi.2011.12.009)

18 Hickmott L, De La Pena H, Turner H, Ahmed F, Protheroe A, Grossman A \& Gupta A. Anti-PD-L1 atezolizumab-induced autoimmune diabetes: a case report and review of the literature. Targeted Oncology 201712 235-241. (https://doi.org/10.1007/s11523017-0480-y) 
19 Deshpande P, Lucas M, Brunt S, Lucas A, Hollingsworth P \& Bundell C. Low level autoantibodies can be frequently detected in the general Australian population. Pathology 201648 483-490. (https://doi.org/10.1016/j.pathol.2016.03.014)

20 Joshi MN, Whitelaw BC, Palomar MT, Wu Y \& Carroll PV. Immune checkpoint inhibitor-related hypophysitis and endocrine dysfunction: clinical review. Clinical Endocrinology 201685 331-339. (https://doi.org/10.1111/cen.13063)

21 Faje A, Sullivan R, Lawrence D, Tritos N, Fadden R, Klibanski A \& Nachtigall L. Ipilimumab-induced hypophysitis: a detailed longitudinal analysis in a large cohort of patients with metastatic melanoma. Journal of Clinical Endocrinology and Metabolism 201499 4078-4085. (https://doi.org/10.1210/jc.2014-2306)

22 Albarel F, Gaudy C, Castinetti F, Carre T, Morange I, Conte-Devolx B, Grob J \& Brue T. Long term follow-up of ipilimumab-induced hypophysitis, a common adverse event of the anto-CTLA-4 antibody in melanoma. European Journal of Endocrinology 2015172 195-204. (https://doi.org/10.1530/EJE-14-0845)

23 Min L, Hodi F, Globble-Hurder A, Ott P, Luke J, Donahue H, Davis M, Carroll R \& Kaiser U. Systemic high-dose corticosteroid treatment does not improve the outcome of ipilimumab-related hypophysitis: a retrospective cohort study. Cancer Research 201421 749-755. (https://doi.org/10.1158/1078-0432.CCR-14-2353)
24 Juszczak A, Gupta A, Karavitaki N, Middleton MR \& Grossman AB. Ipilimumab: a novel immunomodulating therapy causing autoimmune hypophysitis: a case report and review. European Journal of Endocrinology 2012167 1-5. (https://doi.org/10.1530/EJE12-0167)

25 Faje A. Immunotherapy and hypophysitis: clinical presentation, treatment, and biologic insights. Pituitary 201619 82-92. (https:// doi.org/10.1007/s11102-015-0671-4)

26 Chodakiewitz Y, Brown S, Boxerman JL, Brody JM \& Rogg JM. Ipilimumab treatment associated pituitary hypophysitis: clinical presentation and imaging diagnosis. Clinical Neurology and Neurosurgery 2014125 125-130. (https://doi.org/10.1016/j. clineuro.2014.06.011)

27 Di Giacomo A, Biagioli M \& Maio M. The emerging toxicity profiles of anti-CTLA-4 antibodies across clinical indications. Seminars in Oncology 201037 499-507. (https://doi.org/10.1053/j. seminoncol.2010.09.007)

28 Lammert A, Schneider H, Bergmann T, Benck U, Kramer B, Gartner R, Metzner C, Schofl C \& Berking C. Hypophysitis caused by ipilimumab in cancer patients: hormone replacement or immunosuppressive therapy. Experimental and Clinical Endocrinology and Diabetes 2013121 581-587. (https://doi.org/10.105 5/s-0033-1355337)

Received 3 October 2017

Revised version received 24 November 2017

Accepted 28 November 2017 